\title{
LOCOMOTION IN APLYSIA: TRIGGERING BY SEROTONIN AND MODULATION BY BAG CELL EXTRACT ${ }^{1}$
}

\author{
STEVE MACKEY² AND THOMAS J. CAREW
}

Center for Neurobiology and Behavior, Departments of Psychiatry and Physiology, College of Physicians and Surgeons, Columbia University, and New York State Psychiatric Institute, New York, New York 10032

Received September 24, 1982; Revised February 22, 1983; Accepted March 3, 1983

\begin{abstract}
Locomotion in Aplysia is mediated by a central program. We have found that this program is triggered by the biogenic amine, serotonin $(5-\mathrm{HT})$, and modulated by an endogenous source of neuropeptides, bag cell extract.

In examining the triggering of locomotion, we found that injecting 5-HT into the bloodstream of quiescent animals elicited locomotion in both intact, freely moving animals and split-foot preparations. Increasing the concentration of 5-HT from $10^{-7}$ to $10^{-3} \mathrm{M}$ produced both a monotonic increase in the number of steps elicited over a 5-min period after injection and a monotonic decrease in the first interstep interval. The threshold for triggering locomotion was $10^{-7} \mathrm{M}$ in split-foot preparations. Application of a saturated salt solution to the tail-a natural trigger for escape locomotionfacilitated 5-HT locomotion. The effects of 5-HT on locomotion were relatively specific since they were not mimicked by three other biogenic amines known to exist in Aplysia (dopamine, octopamine, and histamine). 5-HT could still trigger locomotion after isolating the pedal and pleural ganglia from the rest of the central nervous system, suggesting that a primary site for the action of 5-HT on locomotion lies in the pedal and pleural ganglia.

In examining the modulation of locomotion, we found that injecting bag cell extract, an endogenous source of neuropeptides in Aplysia which elicits egg-laying behavior, reversibly suppressed 5-HTtriggered locomotion. As in the case with serotonin, bag cell extract suppression of locomotion was still observed in animals in which the pleural and pedal ganglia were isolated from the rest of the central nervous system. The suppression of locomotion was specific to bag cell extract since no suppression was produced by extracts of other central nervous tissue (cerebral ganglia). Moreover, the suppressive effect of bag cell extract was abolished by prior treatment of the extract with pronase, suggesting that the active suppressive agent is one or more of the peptides specific to the bag cells. Thus, locomotion in Aplysia provides a useful model system for examining both the triggering and the modulation of a fixed action pattern.
\end{abstract}

Locomotion in Aplysia californica is a centrally programmed behavioral sequence which is utilized by the animal to accomplish a number of different behavioral objectives. Thus it is integrated with many other components of the animal's behavioral repertory. As a result, locomotion can be elicited and modulated by a variety of

\footnotetext{
${ }^{1}$ This work was supported by National Institute of Neurological and Communicative Disorders and Stroke Grant NS12744, a Schepp Foundation Scholarship to S. M., and Career Development Award MH 0081 to T. J. C. We wish to thank E. R. Kandel for his many helpful discussions and for his useful criticisms of an earlier draft of the manuscript, U. Koch and J. Koester for their assistance in the in vivo preparations, and $\mathrm{K}$. Weiss for his suggestions concerning pronase treatment of bag cell extract.

${ }^{2}$ To whom correspondence should be addressed, at Center for Neurobiology and Behavior, New York State Psychiatric Institute-Annex, 722 West 168 Street, New York, NY 10032.
}

factors including appetitive stimuli (Preston and Lee, 1973; Kupfermann, 1974; Advokat et al., 1978), noxious stimuli (Wachtel and Impelman, 1973; Walters et al., 1978), reproductive behavior (Strumwasser et al., 1969, 1980; Arch, 1976), and both nonassociative and associative learning (Walters et al., 1979, 1981). Moreover, locomotion in Aplysia is well suited for a cellular analysis (Jahan-Parwar and Fredman, 1978a, b, c; Henning et al., 1979). Thus, this fixed action pattern is an excellent system to explore the neural mechanisms involved in the triggering and modulation of a complex behavior. As a first step in this direction, we have examined the triggering effects of several biogenic amines and the modulating effects of bag cell extract (BCE), which contains at least four neuropeptides, including egg-laying hormone (Loh et al., 1975; Arch et al., 1976a, b; Stuart et al., 1980; Scheller et al., 1982). We found that micromolar injec- 
tions of the biogenic amine, serotonin (5-HT) into the bloodstream of non-locomoting animals triggers locomotion. This effect seems to be specific to 5 -HT because it is not mimicked by other biogenic amines known to exist in Aplysia. We have found that 5-HT is likely to exert its primary action on neurons in the pedal and pleural ganglia since it can still elicit locomotion in preparations in which these ganglia have becn surgically isolated from the rest of the central nervous system (CNS).

We have also examined the modulating effects on 5HT-triggered locomotion of an endogenous source of several neuropeptides in Aplysia, BCE, which triggers egg-laying behavior (Kupfermann, 1967). We have found that injections of $\mathrm{BCE}$ prior to injections of $5-\mathrm{HT}$ reversibly suppress 5 -HT-triggered locomotion. This suppressive effect is observed both in animals with intact nervous systems and in animals in which the pedal and pleural ganglia are isolated from the rest of the CNS but are connected to the periphery. Furthermore, the suppressive effect of BCE is abolished by prior treatment of the extract with pronase, suggesting that the active agent in the extract is a neuropeptide. Thus, locomotion in Aplysia provides a useful model system for examining triggering and modulation of a complex fixed action pattern on both behavioral and cellular levels.

Preliminary results of some of the experiments described in this paper have been reported previously in abstract form (Mackey et al., 1981).

\section{Materials and Methods}

In our experiments we have used two types of preparations: (1) intact, freely moving animals with a cannula chronically implanted in their anterior aorta, and (2) split-foot preparations whose CNSs are completely exposed.

Intact animals. In six animals, the anterior aorta was exposed, and a T-shaped cannula (1-mm internal diameter) was inserted into its lumen and tied at both ends (Koch and Koester, 1982). Each animal was first injected with $25 \%$ of its body weight of isotonic $\mathrm{MgCl}_{2}$. A 3- to 4$\mathrm{cm}$ incision was made on the side of the foot at the junction with the legument and body wall, starting at the caudal end of the pro-segment. A 15-cm length of Silastic tubing $(0.5-\mathrm{mm}$ internal diameter) was attached to the long arm of the $\mathrm{T}$ and passed through the body wall, allowing substances to be directly injected into the artery through the Silastic tubing. The Silastic tubing was kept filled with artificial sea water (ASW) and plugged at its free end to prevent blood loss and to maintain normal blood pressure. The volume of the dead space was approximately $0.2 \mathrm{ml}$. The incision was sutured closed and the animal's initial body weight was restored with an injection of ASW into the hemocoel. The animal was allowed to recover for at least $24 \mathrm{hr}$.

Split-foot preparations. Behavioral studies were also performed using a modified split-foot preparation (Hening et al., 1979). Animals weighing between 150 and 300 gm were anesthetized with $\mathrm{MgCl}_{2}$ and pinned dorsal side up in a Lucite chamber filled with isotonic $\mathrm{MgCl}_{2}$ solution. The viscera and buccal mass were excised and the anterior end of the anterior aorta was tied off. A longi- tudinal incision was made down the middle of the foot from the propodium to the tail, approximately threefourths and the length of the foot. The $\mathrm{MgCl}_{2}$ solution was removed and replaced with ASW. The anterior aorta was cannulated and perfused with aerated ASW. The cannula was equipped with a diaphragm that allowed direct injections of substances into the bloodstream. The duration of injection was approximately $5 \mathrm{sec}$; transit time from injection to arrival at the CNS was approximately $15 \mathrm{sec}$. In some experiments either the pedalcerebral, pleural-cerebral, and pleural-abdominal connectives and commissure or the nerves of the pedal and pleural ganglia were cut; otherwise the circumesophageal ganglion and the peripheral nerves were left intact. All animals were perfused with normal ASW for at least $1 \mathrm{hr}$ before testing. The arterial perfusion rate was maintained at approximately $1.5 \mathrm{ml} / \mathrm{min}$ throughout the experiment.

Locomotion was recorded with three Grass FT 0.03 strain gauges attached by elastic threads to stainless steel hooks which, in turn, were hooked through silk suture loops sewn into the foot. The sutures were placed at three sites along the margin of the podium and tegument: two at bilaterally symmetrical sites on the right and left side of the pro-segment and a third on the right side at the meso- or meto-segment. This arrangement allowed us to distinguish true "steps" during locomotion from other types of contractions that were not part of the locomotor pattern, since normal steps (1) occur bilaterally at homologous sites on the foot and (2) consist of a monotaxic wave that progresses in a retrograde direction along the foot (Jahan-Parwar and Fredman, 1978a; Hening et al., 1979).

Bag cell and control extracts. Animals weighing between 250 and $500 \mathrm{gm}$ were sacrificed, and either bag cells or cerebral ganglia were removed. The bag cell clusters or cerebral ganglia (used for control extracts) from an individual animal were placed in $0.3 \mathrm{ml}$ of ASW and homogenized by hand with glass pestles in glass homogenizers, and then were kept on ice. Each homogenate was transferred to centrifuge tubes, and an additional $1.2 \mathrm{ml}$ of buffer was used to rinse out the homogenizer and was transferred to the homogenate. The extract was centrifuged at $10^{4} \mathrm{rpm}$ for $10 \mathrm{~min}$ at $4^{\circ} \mathrm{C}$ using a Beckman centrifuge. The supernatant fluid was pooled and frozen at $-20^{\circ} \mathrm{C}$ until used. Each injection contained the equivalent of the bag cell clusters or cerebral ganglia of a single animal. Samples were used within $30 \mathrm{~min}$ of thawing.

Pronase treatment. Proteolytic treatment of BCE was carried out with pronase $(90 \mathrm{units} / \mathrm{ml}$ ) based on a modification of a technique used by Kupfermann and Weiss (1976). The pronase was incubated for $30 \mathrm{~min}$ to destroy any contaminating enzyme activities. BCE was prepared as described above. After centrifugation the extract was divided into control and experimental aliquots of $0.75 \mathrm{ml}$ each. Pronase $(0.75 \mathrm{ml})$ was added to the experimental sample, and $0.75 \mathrm{ml}$ of ASW buffer was added to the control and allowed to incubate. After incubation, $75 \mu \mathrm{l}$ of $2 \mathrm{~N} \mathrm{HCl}$ were added to each sample to eliminate enzymatic activity. The samples were then centrifuged for $10 \mathrm{~min}$ at low speed and the supernatant was removed. Tris buffer was added to each supernatant to return the 
$\mathrm{pH}$ to 7.8. Both control and experimental samples contained the equivalent of bag cell from a single animal.

Statistical analysis. All statistical tests were carried out by means of a $t$ test for either correlated means (within-group comparison) or independent means (between-group comparison). All comparisons were twotailed unless otherwise stated. All errors are reported as standard errors of the mean (SEM).

\section{Results}

5-HT elicits locomotion in intact animals. Since the biogenic amine serotonin (5-HT) has a prominent role in both defensive and appetitive arousal responses (Weiss and Kupfermann, 1978; Kandel, 1979), we first examined the effect of 5-HT on locomotion in quiescent animals. We used six freely moving animals with surgical implants that allowed direct injection of 5-HT into the anterior aorta. We first recorded locomotion over a 5 -min period after an injection of $1 \mathrm{ml}$ of ASW to serve as a base line control for injection per se. The duration of injection was approximately $30 \mathrm{sec}$. We then injected into the anterior aorta increasing concentrations of $5-\mathrm{HT}$, from $10^{-7}$ to $10^{-3} \mathrm{M}$ ( $\mathrm{pH}$ of all solutions, 7.8). The duration of injection was approximately $30 \mathrm{sec}$. Each injection was separated by $10 \mathrm{~min}$. The threshold concentration for triggering locomotion ranged from $10^{-6}$ to $10^{-4} \mathrm{M}$ with $50 \%$ of the animals locomoting after a $10^{-5} \mathrm{M}$ injection. These concentrations refer to the concentration of the 1-ml injection. The effective concentration was probably much less, due to dilution by the hemolymph. 5-HT elicited significantly greater locomotion (as measured by the number of steps in a 5-min period; see Walters et al., 1979) than did ASW in all animals $(p<0.02)$. In fact, ASW elicited locomotion in only one animal, and 5-HT in that animal triggered a greater number of steps. Thus, 5-HT, in effective concentrations in the micromolar range, can trigger locomotion in intact, freely moving animals.

5-HT elicits locomotion in split-foot preparations in a dose-dependent fashion. We next extended our study of the effect of 5-HT on locomotion in a modified splitfoot preparation, a simplified preparation in which reliable replicas of locomotion can be observed (Hening et al., 1979; Fig. 1A). The split-foot preparation simplifies the screening of neuroactive substances and is also amenable for subsequent cellular studies. During locomotor episodes the foot exhibits rhythmic patterns of contraction, as observed in freely moving animals, with waves beginning at the anterior margin of the foot and moving posteriorly (Jahan-Parwar and Fredman, 1978a, b, c; Hening et al., 1979). We recorded steps with strain gauges attached to the foot (see "Materials and Methods"). In all experiments, we used to total number of steps exhibited in a 5-min test period following injection of a substance as a measurement of the triggering effects of that substance. To distinguish between triggered and spontaneous locomotion, only steps occurring within 60 sec of injection and with less than a 60 -sec interstep interval were counted as triggered locomotion.

Using split-foot preparations, we first recorded locomotion over a 5 -min period in previously non-locomoting animals after an injection of ASW to serve as a control for the effects of injection per se. We then injected increasing concentrations of 5- HT, from $10^{-7}$ to $10^{-3} \mathrm{M}$, into the anterior aorta. The effective concentration was probably much less, due to dilution in the cannula and anterior aorta. Injections were separated by $10 \mathrm{~min}$ or until the animal stopped locomoting for $10 \mathrm{~min}$ after a prior injection. An example of 5-HT-triggered locomotion is shown in Figure $1 B$, and the relationship between 5$\mathrm{H}^{\prime} \mathrm{T}$ concentration and number of steps is shown in Figure $2 A$. After an injection of $10^{-7}$ м 5-HT, $50 \%$ of the animals $(N=4)$ locomoted within $60 \mathrm{sec}$ of injection. The mean number of steps was $1.5 \pm 0.87$ as compared to control injections of ASW, which produced no locomotion in any of the animals tested. Increasing the concentration of 5HT injected from $10^{-7}$ to $10^{-3} \mathrm{M}$ caused a monotonic increase in number of steps, reaching a mean of $25.0 \pm$ 5.67 steps after an injection of $10^{-3} \mathrm{M} 5-\mathrm{HT}$. Moreover, increasing the concentration of 5-HT produced a monotonic decrease in the first interstep interval, ranging from a mean of $24.5 \pm 13.5 \mathrm{sec}$ after $10^{-7} \mathrm{M} 5-\mathrm{HT}$ to a mean of $8.8 \pm 1.46 \mathrm{sec}$ after $10^{-3}$ м 5 -HT (Fig. $2 B$ ). Thus, with increasing $5-\mathrm{H}$ T concentration, animals not only showed more locomotion but also a faster initial rate of locomotion.

We next examined how locomotion triggered by $5-\mathrm{HT}$ interacts with locomotion triggered by salt, a natural stimulus of escape locomotion. There were three parts to the experiment. (1) We first injected $10^{-4} \mathrm{M} 5-\mathrm{H}^{\prime} \mathrm{T}$ and measured locomotion over a 5 -min period to establish a base line. (2) We then administered a second injection of 5 -HT 20 min later, but immediately prior to this injection, $5 \mathrm{ml}$ of a saturated salt solution was applied to the tail. (3) Finally, after a 20 -min interval, we applied $5 \mathrm{ml}$ of saturated salt solution to the tail in the absence of $5-\mathrm{HT}$, and locomotion was recorded over a 5-min period. The locomotor effects of salt and 5-HT were additive (Fig. 3). The mean amount of 5-HT-triggered locomotion was 14.2 \pm 4.2 steps $(N=6)$. Salt solution alone elicited $7.0 \pm 2.9$ steps. Salt followed by 5 -HT elicited $21.7 \pm 3.3$ steps. The locomotion elicited by salt alone was qualitatively similar to that triggered by $5-\mathrm{HT}$ at a concentration of $10^{-5}$ to $10^{-4} \mathrm{M}$.

Other biogenic amines do not trigger locomotion. To examine the specificity of $5-\mathrm{HT}$, we tested the triggering effects of three other biogenic amines known to exist in Aplysia: dopamine, octopamine, and histamine. Injections of each amine were tested in non-locomoting splitfoot animals ( $N=3$ for each amine tested) and compared with the response to an equivalent concentration of 5$\mathrm{HT}$ in the same animal. We found that injections of from $10^{-4}$ to $10^{-3} \mathrm{~m}$ of dopamine and octopamine failed to elicit any locomotion in any of the animals tested, while $10^{-3}$ M histamine elicited only a slight degree of locomotion (2.3 \pm 2.3$)$ compared to $10^{-3} \mathrm{M} 5$-H' $(34.7 \pm 4.3)$. These results indicate that of four biogenic amines tested, triggering of locomotion appears to be relatively specific to serotonin (Fig. 4).

Previous work has shown that Aplysia contains at least five different receptors to 5-HT (Gerschenfeld and Paupardin-Tritsch, 1974). At least one class of receptors is coupled to adenylate cyclase (Cedar and Schwartz, 1972; Brunelli et al., 1976; Weiss et al., 1978). We were interested to see whether 5-HT-triggered locomotion 

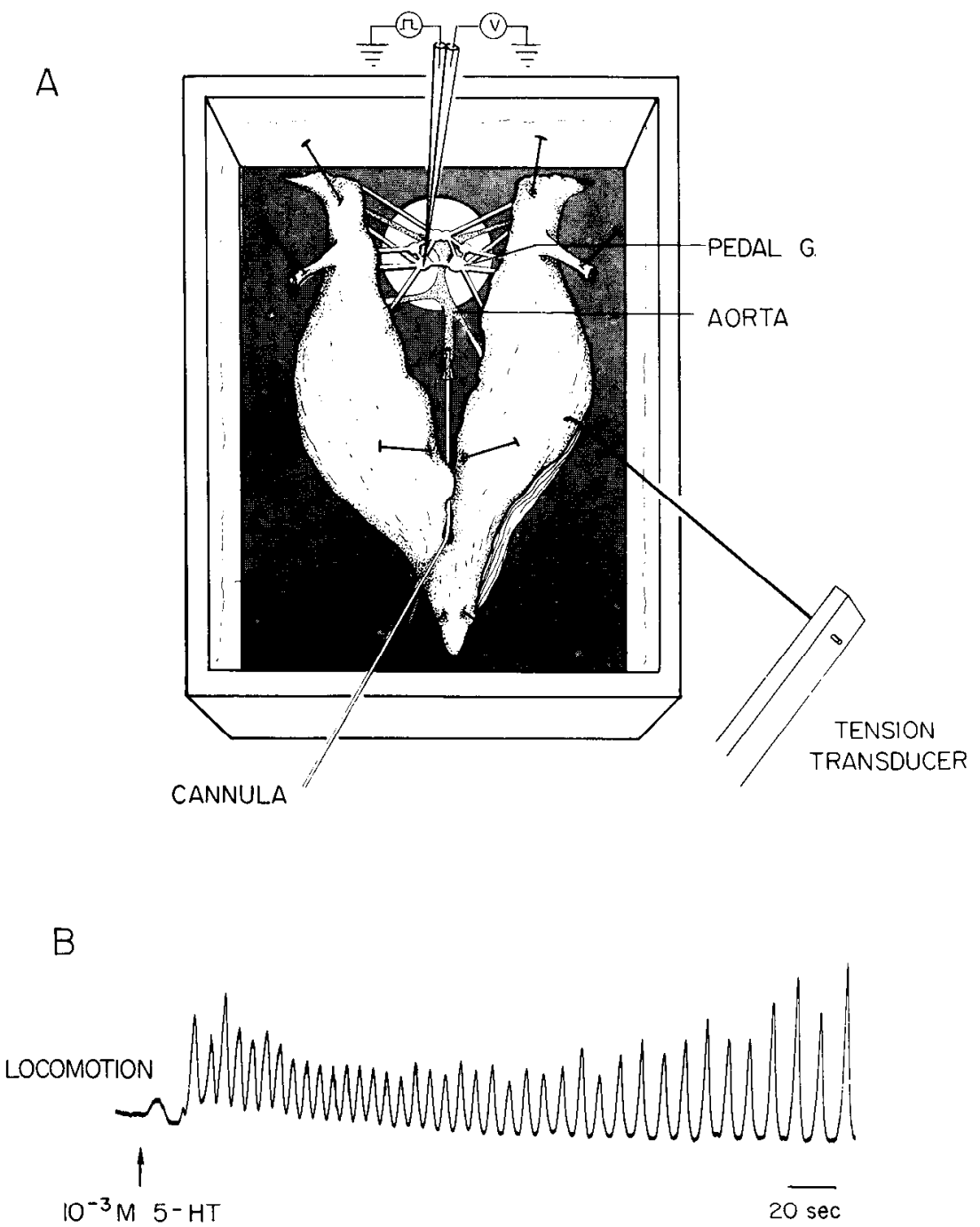

Figure 1. Locomotion is triggered by 5-HT in a split-foot preparation. A, Diagrammatic view of the split-foot preparation. The animal is split down the middle leaving the CNS attached to the periphery (see Hening et al., 1979). The anterior aorta is cannulated and perfused continuously with ASW. This preparation exhibits reliable replicas of locomotion. $G$., ganglion. $B$, A strain gauge record of locomotion triggered by $1 \mathrm{ml}$ of $10^{-3} \mathrm{M} 5$-HT injected into the anterior aorta. Upward deflections reflect forward extension of the foot.

could also be mimicked by a cAMP analogue-benzylthio-cAMP. Previous work in Aplysia has shown that millimolar concentrations of benzylthio-cAMP can simulate cAMP-mediated effects produced by serotonin receptors that are coupled to adenyl cyclase (Weiss et al., 1979). Injections of up to to $10^{-3} \mathrm{M}$ benzylthio-cAMP failed to elicit locomotion in any animals tested $(N=5)$. Moreover, preliminary experiments suggest that cinnanserin-a pharmacological blocker of 5-HT receptors coupled to adenyl cyclase (Brunelli et al., 1976) - has no effect on 5-HT-triggered locomotion. Thus our results suggest that 5 -HT-triggered locomotion is not mediated by cAMP-coupled receptors.

Site of action of 5-HT. The central program for locomotion is located in the circumesophageal ring ganglia which consist of paired cerebral, buccal, pleural, and pedal ganglia. The majority of foot and body wall motor neurons are located in the pedal and pleural ganglia (Hening et al., 1979; Fredman and Jahan-Parwar, 1980). We wanted to determine the site of action of 5-HT. As a first step, we examined whether 5-HT-triggered locomotion could be elicited in a single pedal and an adjacent pleural ganglion isolated from the rest of the circumesophageal ganglia. To accomplish this we cut the pedal commissures and the connectives that run between the cerebrobuccal and the pleural-pedal ganglia (see Fig. 5, inset). Before lesioning, spontaneous locomotion was often observed, but after lesioning, only weak pedal waves were observed. However, injections of $10^{-4} \mathrm{M} 5$ - $\mathrm{HT}$ into the aorta still elicited locomotion qualitatively similar to (and not significantly different from) locomotion elicited before the lesions. In the four animals tested, 5-HT elicited a mean of $17.5 \pm 2.4$ steps after isolating the pedal-pleural ganglia compared to $22.8 \pm 4.91$ steps be- 


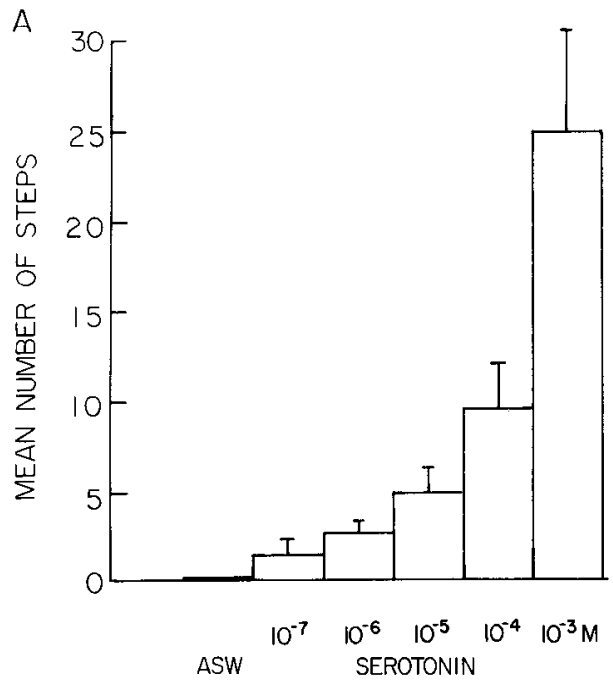

Figure 2. 5-HT-triggered locomotion is concentration dependent. $A$, Increasing concentrations of 5 -HT (serotonin) produce a monotonic increase in the number of steps recorded during a 5-min period following injection ( $N=5$ for ASW and all concentrations of 5-HT except $10^{-7} \mathrm{M}$, where $N=4$ ). Threshold concentration is $10^{-7} \mathrm{M}$. $B$, Increasing concentrations of 5-HT also produce a monotonic decrease in the interstep interval of the first two steps.

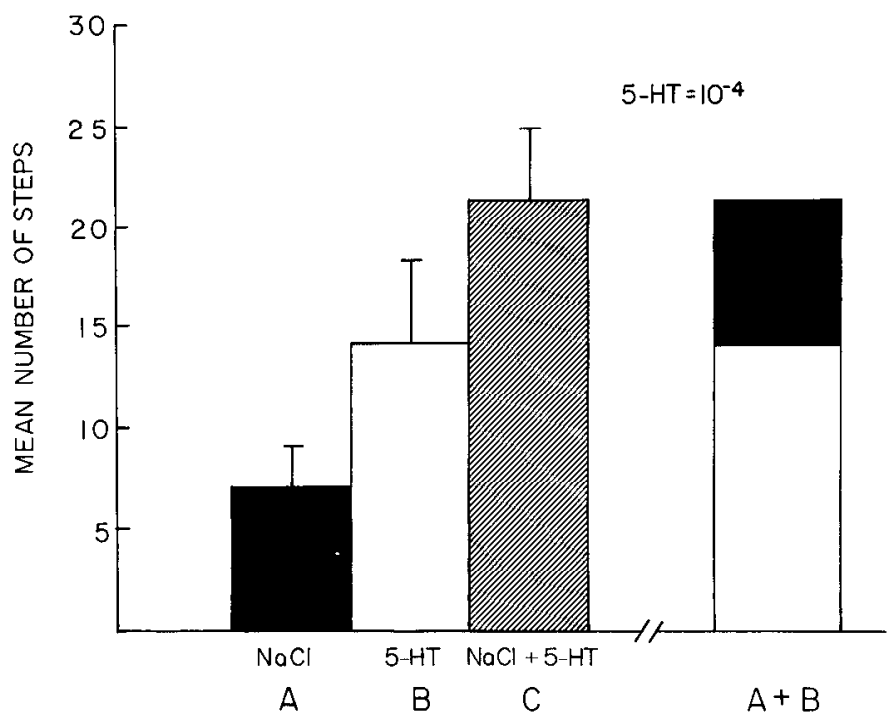

Figure 3. The locomotor effects of a natural trigger for locomotion (salt) and 5-H'T are additive. Three episodes of triggered locomotion are shown $(N=3), A$, Salt-triggered locomotion alone $(0.5 \mathrm{ml}$ of salt solution). $B$, Locomotion triggered after injection of $10^{-4}$ м 5-HT. $C$, Locomotion triggered by a $0.5-\mathrm{ml}$ salt solution applied to the tail, followed by injection of $10^{-4} \mathrm{M} 5-\mathrm{HT}$. As shown on the right $(A+B)$, the locomotor effects of salt and 5-HT are additive.

fore the lesion (Fig. 5). Cutting the pedal nerves from the right pedal ganglion abolished 5-HT-elicited locomotion on that side. These results suggest that a locus for 5-HTelicited locomotion lies within the pedal and pleural ganglia. 5-HT does not trigger locomotion in peripheral neurons or muscles that have been isolated from the CNS, nor does it require the presence of the cerebral or other central ganglia. More recently, we have obtained

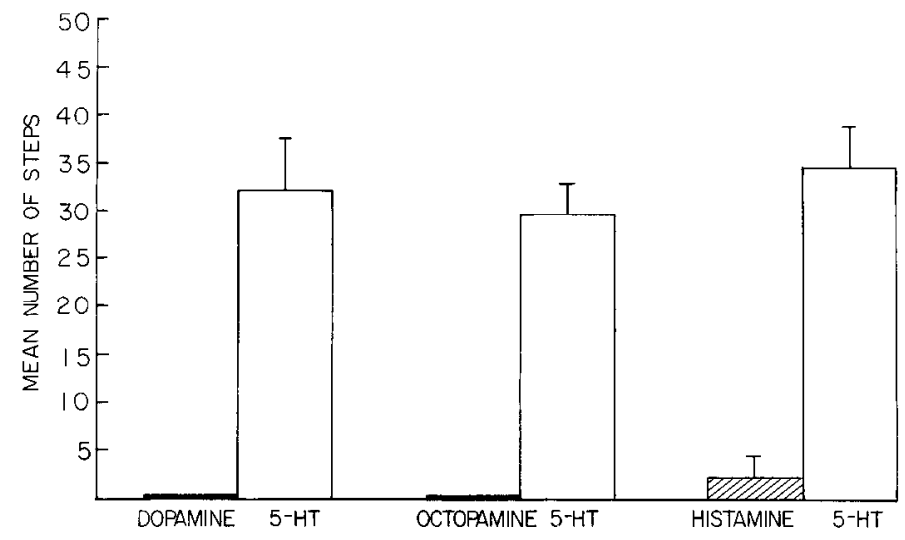

Figure 4. 5-HT-triggered locomotion is specific to 5-HT. Locomotion was recorded after a $1-\mathrm{ml}$ injection of $10^{-3} \mathrm{M}$ dopamine ( $N=3$; left solid har), octopamine $(N=3$; middle solid bar $)$, or histamine $(N=3$; diagonal line bar $)$. Dopamine and octopamine failed to produce any locomotion, while histamine produced only a slight effect. In each case, 5-HT (open bars) produced significantly more locomotion than did the other biogenic amine being tested.

preliminary evidence that locomotion can also be triggered in an isolated pedal ganglion connected only to the foot.

Effects of BCE on 5-HT-triggered locomotion. Egg laying in Aplysia is triggered by BCE, which contains a family of neuropeptides including egg-laying hormone, a peptide of approximately $4400 M_{\mathrm{r}}$, produced by two clusters of bag cells-approximately 400 cells/cluster-located in the abdominal ganglion (Kupfermann, 1967; Strumwasser et al., 1969; Arch, 1972a, b). Injections of BCE into intact, sexually mature animals induces egg laying within $1 \mathrm{hr}$ after injection (Kupfermann, 1967; Strumwasser et al., 1969; Arch, 1976). Egg laying is pre- 

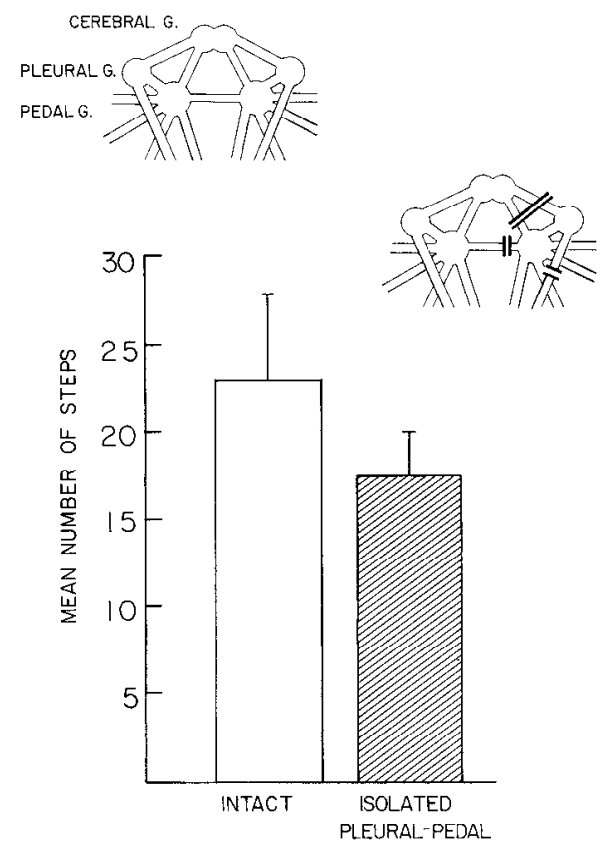

Figure 5. The pedal and pleural ganglia are sites of 5-HT action. After cutting the pedal commissures and the connectives from the pedal-cerebral, pleural-cerebral, and pleural-abdominal ganglia ( $G$.) (see inset), thus isolating the pleural and pedal ganglia from the rest of the CNS, 5-HT still triggers significant locomotion (shaded bar).

ceded by a stereotypic behavioral sequence including buccal protraction, head waving, and a decrease in spontaneous locomotion (Strumwasser et al., 1969; Arch, 1976). We were therefore interested to see whether $\mathrm{BCE}$ could suppress locomotion triggered by 5 -HT. In control animals $(N=7)$, we injected $10^{-4}$ м 5-H T four times at 20-min intervals to assess the effects of repeated 5-HT administration. Over the four test periods there was a slight but progressive tendency for repeated 5-HT applications to produce fewer steps (Fig. 6, control). Experimental animals $(N=5)$ received the same treatment as controls except that they received a $1-\mathrm{ml}$ injection of BCE prior to the second 5-HT injection. These animals showed a mean decrease of $52 \pm 19.2 \%$ in total number of steps after the second injection of $5-\mathrm{HT}$, which was a significantly greater reduction $(p<0.003)$ than that exhibited by the control group (mean decrease $=6 \pm$ $8.8 \%$ ). Moreover, unlike the control group, the group receiving $\mathrm{BCE}$ tended to increase locomotion after the third and fourth injections of 5-HT (returning toward base line levels), suggesting that the effect of BCE was gradually wearing off.

We next determined whether the suppressive effect of BCE could be demonstrated in pedal and pleural ganglia isolated from the rest of the CNS. After isolating the pedal and pleural ganglia, we compared repeated injections of 5-HT in control animals to repeated injections of $5-\mathrm{HT}$ with an injection of BCE preceding the second injection of 5-HT. Application of BCE still caused a significant reduction in 5-HT-triggered locomotion $(p<$ 0.05 , one-tailed test; Fig. 7). Thus, these results suggest that a site of BCE modulation of 5-HT-triggered locomotion is located in the pleural and pedal ganglia.

To show that the supression of 5-HT-triggered loco- motion was specific to the BCE, we also examined the effect of extracts of other nervous tissue (cerebral ganglia) in 10 animals using the same experimental procedure as above. Cerebral extracts had no suppressive effects on locomotion; they produced results that were comparable to control injections of ASW.

Previous studies have shown that BCE contains at least four neuropeptides including ELH (egg-laying hormone) (Loh et al., 1975; Arch et al., 1976a, b; Stuart and Strumwasser, 1980; Scheller et al., 1982). Moreover, Toevs and Brackenbury (1969) have shown that the egglaying efficacy of BCE is sensitive to pronase treatment. To examine whether the suppression of 5-HT-elicited locomotion is produced by a peptide in the BCE, we subjected aliquots of BCE to pronase digestion (see "Materials and Methods"). The results of our experi-

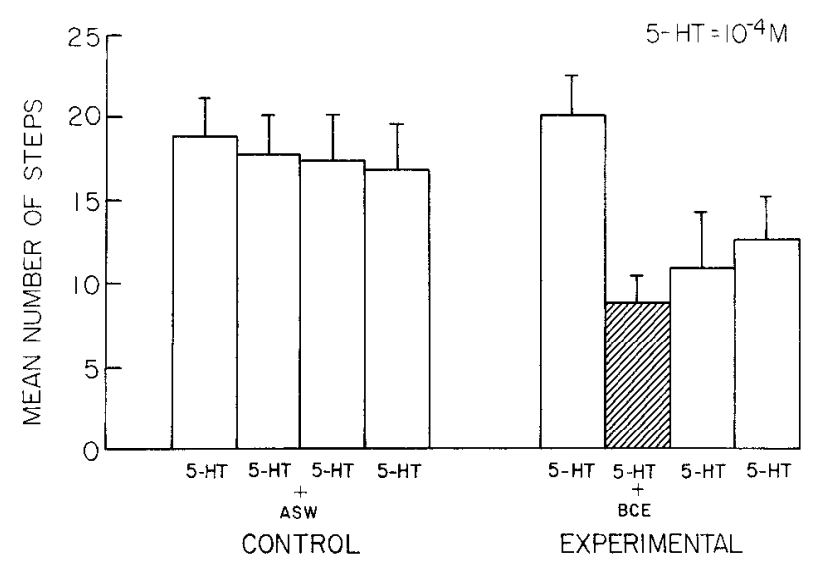

Figure 6. BCE (containing at least four neuropeptides) suppresses 5-HT-triggered locomotion. Left, Four control injections of 5 -HT separated by $20 \mathrm{~min}$ produce a slight but progressive decrement in locomotion $(N=7)$. Right, In five experimental preparations, injection of $1 \mathrm{ml}$ of $\mathrm{BCE}$ prior to the injection of 5-HT significantly suppresses 5-H'T-triggered locomotion $(N=5, p<0.003)$. The suppression is specific to bag cells: injection of extracts of other CNS regions (e.g., cerebral ganglia) do not produce suppression.

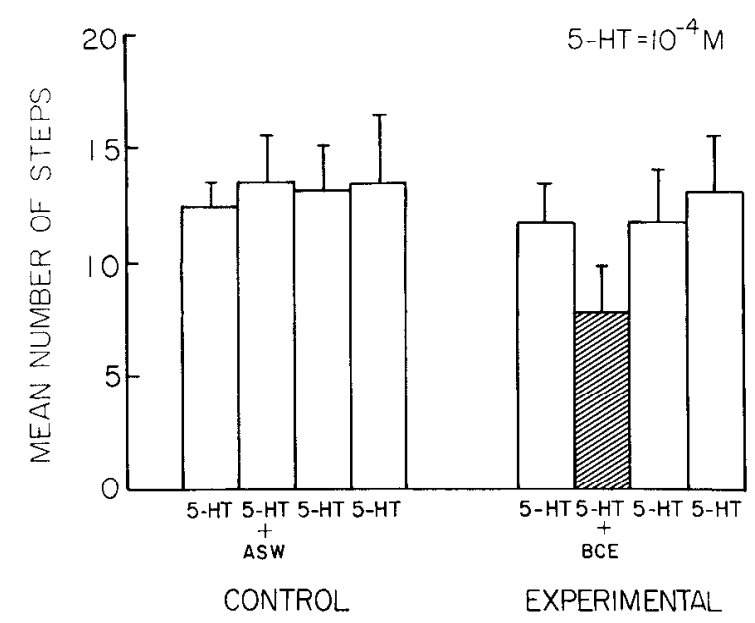

Figure 7. The pedal and pleural ganglia are sites of BCE suppression of 5-HT-triggered locomotion. The pedal commissures and the pedal and pleural connectives were cut isolating the pleural and pedal ganglia (as in Fig. 5). Using the same protocol as in the intact CNS (see Fig. 6), BCE still reversibly suppresses 5-HT-triggered locomotion. 


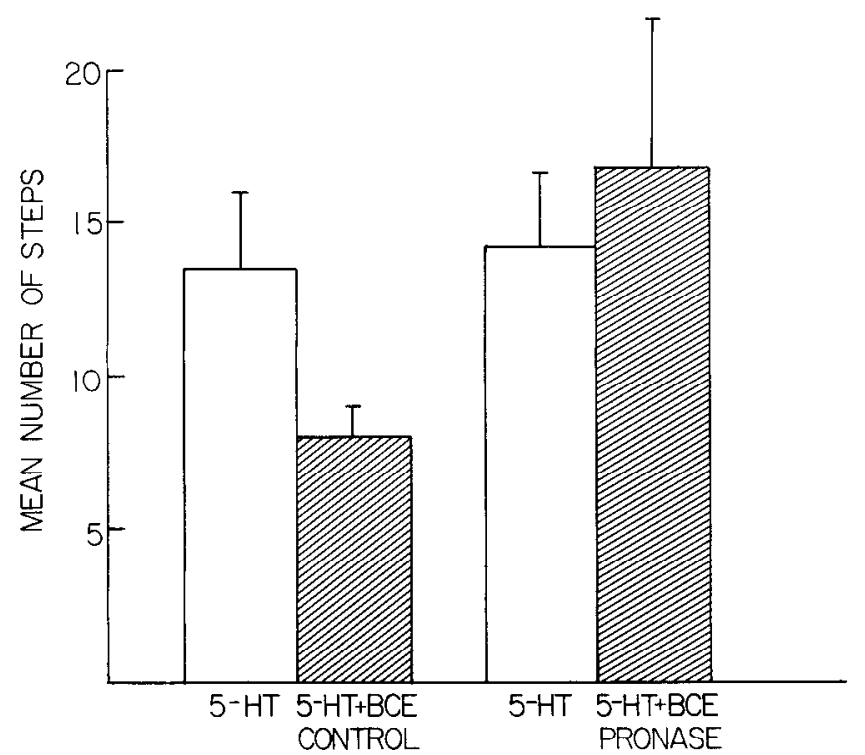

Figure 8. BCE suppression of 5-HT-triggered locomotion is blocked by pronase treatment. Left, BCE (control, $N=2$ ) suppresses 5-HT-triggered locomotion. Right, BCE treated with pronase $(N=3)$ no longer produces suppression, suggesting that the active agent in the $\mathrm{BCE}$ is one of the peptides synthesized by bag cells.

ments are shown in Figure 8. The suppressive effect of $\mathrm{BCE}$ on 5-HT-triggered locomotion is completely blocked by prior treatment of the extract with pronase. These results thus suggest that the active agent in the $\mathrm{BCE}$ is likely to be one of the neuropeptides released by bag cells.

\section{Discussion}

Studies in a variety of vertebrate and invertebrate systems have shown that many cyclic behaviors (locomotion, feeding, scratching) are centrally programmed (for review see Grillner, 1975; Stein, 1978). Several important questions have arisen concerning (1) the identity of the neuroactive substances (neurotransmitters or neurohormones) involved in eliciting and modulating these motor programs, (2) the site and mechanism of these transmitters' actions, and (3) the way in which cyclic behaviors are integrated into more complex behavioral sequences (such as avoidance behavior, appetitive behavior, and reproduction). In attempting to answer these questions, considerable attention has recently focused on two classes of neuroactive substances: biogenic amines and hormones-in particular, neuropeptides.

Triggering of central programs by biogenic amines. There is good evidence that several central programs for locomotion can be triggered or facilitated by biogenic amines (Grillner, 1975; Shik and Orlovsky, 1976; Willard, 1980). M. L. Shik and G. N. Orlovsky have examined locomotion in cats with brainstem transections in which the mesencephalon remains intact. Electrical stimulation of a circumscribed area, nucleus cuneiformis, also known as the mesencephalic locomotor region (MLR), evokes locomotion (Shik, 1971). In chronic preparations, maintained stimulation produces continuous locomotion (Sirota et al., 1973). Intravenous injection of phenoxybenzamine (an $\alpha$-adrenergic blocker) blocks MLR-evoked lo- comotion (Grillner, 1973). This suggests that noradrenergic neurons originating in the lower brainstem give rise to one or more descending pathways which mediate MLR-evoked locomotion. Moreover, in acute and chronic spinally injected cats, intravenous injection of 3,4-dihydroxyphenylalanine (DOPA), a precursor of norepinephrine, elicits locomotion similar to that triggered by MLR stimulation. Intravenous injections of clonidine (an $\alpha^{-}$ agonist) also elicit locomotion. Finally, intravenous injection of 5-HT mimics the locomotor effects of DOPA or clonidine and also increases the excitability of motor neurons (Ahlman et al., 1971). The triggering of locomotion by biogenic amines is not limited to vertebrates. Willard (1980) has examined the effect of 5-HT on the program for swimming in the leech. Using an isolated nerve cord preparation, Willard (1980) has shown that swimming programs can be elicited after exposure to $5 \times 10^{-6}$ to $10^{-5} \mathrm{M}$ serotonin in the bathing medium. Using a very small volume chamber, he has also found that stimulation of the Retzius cell, a serotonergic neuron, can trigger locomotion. Finally, injection of $100 \mu \mathrm{g}$ of 5-HT into intact animals increases the occurrence of swimming sequences. Thus, biogenic amines are implicated as possible triggers of locomotion in both vertebrates and invertebrates.

Our studies provide further support for the findings, based both on vertebrates and on the leech, that biogenic amines-in particular, 5 - $\mathrm{H}^{\mathrm{T}}$ - can have a triggering effect on central locomotor programs. In Aplysia, cutting the cerebral-pleural connectives abolishes locomotor activity (Jahan-Parwar and Fredman, 1979). This suggests that cells which trigger locomotion are located in the cerebral ganglia. More recently, Fredman and Jahan-Parwar (1981) have identified neurons in the cerebral ganglia which may constitute a "command system" for locomotion. It will be interesting to determine whether these cerebral neurons might be serotonergic or whether they might stimulate serotonergic followers.

Modulatory effects of hormones and peptides. In the last decade it has become clear that hormone receptors are widely distributed in nervous systems and that in addition to their effects on target organs, hormones have important triggering and modulatory roles in a variety of behaviors. A major principle which has emerged is that the behavioral effects of hormones are often linked to their other physiological actions. For example, Pfaff (1978) has demonstrated in the rat that the sex steroid, estrogen, which has receptors in the reproductive organs, also has receptors in the rat hypothalamus which appear to trigger copulatory behavior (lordosis). Similarly, Arnold et al. (1976) have shown that testosterone, which determines the development of male sexual characteristics, also has a critical role in the development of birdsong in the male finch-an important courting behavior. Finally, Fitzsimmons (1978) and Weindl (1973) have shown that the octapeptide, angiotensin II, which maintains body volume through several physiological mechanisms including increased resorption of $\mathrm{Na}^{+}$and water (via stimulation of the release of aldosterone and vasopressin) and direct vasoconstriction of arteries, also has binding sites in the subfornical organ of the rat brain which induce drinking behavior.

Modulation of neural circuitry by a given class of 
hormones may occur in many regions of the nervous system. Perhaps the best examples are the bag cell neuropeptides, including ELH. Mayeri et al. (1979a, b) have shown that the neurosecretory products of bag cells exert a broad range of changes in the activity of identified neurons which are part of previously defined neuronal circuits in the abdominal ganglia of Aplysia. These include ink motor neurons, the command cell of the cardiovascular system (L10), and motor neurons of the gill and siphon withdrawal reflex. Stuart and Strumwasser (1980) have extended these findings to the buccal ganglia and feeding circuits and have shown that direct application of ELH to the buccal ganglia activates an identified neuron (bn 3 ) which causes retraction of the buccal mass, thus perhaps participating in terminating feeding. This observation is particularly significant in that inhibition of feeding is important prior to the deposition of the egg mass, and it highlights the coordination between the behavioral effects of ELH and its other physiological effects.

As described above, egg-laying behavior has many components that are coordinated into a complex, integrated sequence. This is especially interesting in light of the fact that bag cells, like other neuroendocrine tissue, release not only ELH but at least four peptides (Arch, 1972a, b; Strumwasser et al., 1980; Scheller et al., 1982). Like pro-opiomelanocortin, at least some of these peptides are derived from a common precursor polypeptide that is post-translationally cleaved to yield active molecules. However, unlike pro-opiomelanocortin, which is encoded in a single gene, Scheller et al. (1982) have shown that there are at least five genes in the haploid genome containing ELH sequences. Furthermore, the multigene family yields at least five discrete mRNA transcripts which in turn are processed and transcribed to yield polypeptides. It will be interesting to determine whether some of these distinct peptides are responsible for mediating the different aspects of egg-laying behavior, including the suppression of locomotion.

Our results suggest that $\mathrm{BCE}$, probably through the action of one or more neuropeptides, suppresses locomotion triggered by serotonin. At least one site of this action lies in the pedal-pleural ganglia. In order to deposit its eggs, an animal must cease locomoting. Thus, suppression of locomotion by BCE provides yet another example of the coordination of motivationally consistent behavior by a neuropeptide hormone. Moreover, our results provide a system that is advantageous for studying the interaction of a biogenic amine and neuropeptide(s) on a central motor program. The next step will be to extend our behavioral studies to a cellular level by recording from identified motor neurons involved in locomotion in order to analyze the mechanisms and sites of action of these two classes of neuroactive substances on triggering and modulating of a complex fixed action pattern.

\section{References}

Advokat, C., T. J. Carew, and E. R. Kandel (1976) Modulation of a simple reflex in Aplysia. Soc. Neurosci. Abstr. 2: 513.

Ahlman, H. S., S. Grillner and M. Udo (1971) The effect of 5HTP on the static fusimotor activity and the tonic stretch reflex of an extensor muscle. Brain Res. 17: 393-396.
Arch, S. (1972a) Polypeptide sccretion from the isolated PVG of Aplysia californica. J. Gen. Physiol. 59: 47-59.

Arch, S. (1972b) Biosynthesis of egg laying hormone (ELH) in the bag cell neurons of Aplysia californica. J. Gen. Physiol. 60: $102-119$.

Arch, S. (1976) Neuroendocrine regulation of egg laying in Aplysia californica. Am. Zool. 16: 167-175.

Arch, S., P. Earley, and T. Smock (1976a) Biochemical isolation and physical identification of the egg laying hormone in Aplysia californica. J. Gen. Physiol. 68: 197-210.

Arch, S., T. Smock, and P. Farley (1976h) Precursor and product processing in the bag cell neurons of Aplysia californica. J. Gen. Physiol. 68: 211-225.

Arnold, P., F. Nottebohm, and D. W. Pfaff (1976) Hormone concentrating cells in vocal control and other areas of the brain in the zebra finch, Poephila guttata. J. Comp. Neurol. 165: $487-512$.

Brunelli, M., V. Castellucci, and E. R. Kandel (1976) Synaptic facilitation and behavioral sensitization in Aplysia: Possible role of serotonin and cyclic AMP. Science 194: 1178-1180.

Cedar, H., and J. H. Schwartz (1972) Cyclic adenosine monophosphate in the nervous system of Aplysia californica. II. Effect of serotonin and dopamine. J. Gen. Physiol. 60: 570587.

Fitzsimmons, J. T. (1978) Angiotensin and thirst. Neurosci. Res. Program Bull. 16: 307-313.

Fredman, S. M., and B. Jahan-Parwar (1980) Role of pedal ganglia motor neurons in pedal wave generation in Aplysia. Brain Res. Bull. 5: 179-193.

Fredman, S. M., and B. Jahan-Parwar (1981) Cerebral neurons constituting a command system for locomotion in Aplysia. Soc. Neurosci. Abstr. 7: 642.

Gerschenfeld, H. M., and D. Paupardin-Tritsch (1974) Ionic mechanisms and receptor response underlying the responses of molluscan neurones to 5-hydroxytryptamine. J. Physiol. (Lond.) 243: 427-456.

Grillner, S. (1973) Locomotion in the spinal cat. In Control of Posture and Locomotion, R. B. Stein et al., eds., pp. 515-536, Plenum Press, New York.

Grillner, S. (1975) Locomotion in vertebrates: Central mechanisms and reflex interaction. Physiol. Rev. 55: 247-304.

Hening, W. A., E. T. Walters, T. J. Carew, and E. R. Kandel (1979) Motor neuronal control of locomotion in Aplysia. Brain Res. 179: 231-253.

Jahan-Parwar, B., and S. M. Fredman (1978a) Pedal locomotion in Aplysia. I. Sensory and motor function of foot nerves. Comp. Biochem. Physiol. (A) 60: 459-465.

Jahan-Parwar, B., and S. M. Fredman (1978b) Control of pedal and parapodial movements in Aplysia. I. Proprioceptive and tactile reflexes. J. Neurophysiol. 41: 600-608.

Jahan-Parwar, B., and S. M. Fredman (1978c) Control of pedal and parapodial movements in Aplysia. II. Cerebral ganglion neurons. J. Neurophysiol. 41: 609-619.

Jahan-Parwar, B. and S. M. Fredman (1979) Neural control of locomotion in Aplysia: Role of the central ganglia. Behav. Neural. Biol. 27: 39-58.

Kandel, E. R. (1979) Search for the cellular mechanisms for arousal and sensitization in Aplysia. Neurosci. Res. Program Bull. 17: 545-555.

Koch, U., and J. Koester (1982) Time-sharing of heart power: Cardiovascular adaptations to food-arousal in Aplysia. J. Comp. Physiol. 149: 31-42.

Kupfermann, I. (1967) Stimulation of egg laying: Possible neuroendocrine function of abdominal ganglion of Aplysia californica. Nature 216: 814-815.

Kupfermann, I. (1974) Feeding behavior in Aplysia: A simple system for the study of motivation. Behav. Biol. 10: 1-26.

Kupfermann, I., and K. Weiss (1976) Water regulation by a presumptive hormone contained in identified neurosecretory 
cell R15 of Aplysia. J. Gen. Physiol. 67: 113-123.

Loh, Y. P., Y. Sarne, and H. Gainer (1975) Heterogeneity of proteins synthesized, stored, and released by the bag cells of Aplysia californica. J. Comp. Physiol. 100: 283-295.

Mackey, S., T. J. Carew, and E. R. Kandel (1981) Modulation of the central program for locomotion by serotonin and bag cell extract (egg-laying hormone). Soc. Neurosci. Abstr. 7: 380 .

Mayeri, E., P. Brownell, W. D. Branton, and S. B. Simon (1979a) Multiple, prolonged actions of neuroendocrine bag cells on neurons in Aplysia. I. Effects on bursting pacemaker neurons. J. Neurophysiol. 42: 1165-1184.

Mayeri, E., P. Brownell, and W. D. Branton (1979b) Multiple, prolonged actions of neuroendocrine bag cells on neurons in Aplysia. II. Effects on beating pacemaker and silent neurons. J. Neurophysiol. 42: 1185-1197.

Pfaff, D. W. (1978) Peptide and steroid hormones and the neural mechanisms for female reproductive behavior. In The Hypothalamus, S. Riechlin, R. J. Baldessarini, and J. B. Martin, eds., pp. 245-253, Raven Press, New York.

Preston, R. J., and R. M. Lee (1973) Feeding behavior in Aplysia californica: Role of chemical and tactile stimulation. J. Comp. Physiol. Psychol. 82: 368-381.

Scheller, R. H., J. F. Jackson, L. B. McAllister, J. H. Schwartz, E. R. Kandel, and R. Axel (1982) A family of genes that codes for ELH, a neuropeptide eliciting a stereotyped pattern of behavior in Aplysia. Cell 28: 707-719.

Shik, M. L. (1971) The controlled locomotion of the mesencephalic cat. Proc. Int. Congr. Physiol. 8: 104-105.

Shik, M. L., and G. N. Orlovsky (1976) Neurophysiology of locomotor automatism. Physiol. Rev. 56: 465-501.

Sirota, M. G., T. J. Sirota, and M. L. Shik (1973) The cat locomotion elicited through the electrode implanted in the brain. Sechenov. Physiol. J. USSR. 59: 1314-1321.

Stein, P. J. (1978) Motor systems with specific reference to the control of locomotion. Annu. Rev. Neurosci. 1: 61-82.

Strumwasser, F., J. W. Jacklet, and R. B. Alvarez (1969) A seasonal rhythm in the neural extract induction of behavioral egg laying in Aplysia. Comp. Biochem. Physiol. 29: 197-206.

Strumwasser, F, L. K. Kaczmarek, A. Y. Chiu, E. Heller, K. R. Jennings, and D. P. Viele (1980) Peptides controlling behav- ior in Aplysia. Soc. Gen. Physiol. Ser. 35: 197-218.

Stuart, D. K., A. Y. Chiu, and F. Strumwasser (1980) Neurosecretion of egg laying hormone and other peptides from electrically active bag cell neurons of Aplysia. J Neurophysiol. 43: 488-498.

Toevs, L. A., and R. W. Brackenbury (1969) Bag cell specific proteins and the humoral control of egg laying in Aplysia californica. Comp. Biochem. Physiol. 29: 207-216.

Wachtel, H., and D. Impelman (1973) A galloping escape behavior in Aplysia californica. Fed. Proc. 32: 368.

Walters, E. T., T. J. Carew, and E. R. Kandel (1978) Conflict and response selection in the locomotor system of Aplysia. Soc. Neurosci. Abstr. 4: 209.

Walters, E. 'T., 'T. J. Carew, and E. R. Kandel (1979) Classical conditioning in Aplysia californica. Proc. Natl. Acad. Sci. U. S. A. 76: 6675-6679.

Walters, E. T., T. J. Carew, and E. R. Kandel (1981) Associative learning in Aplysia: Evidence for conditioned fear in an invertebrate. Science 211: 504-506.

Weindl, A. (1973) Neuroendocrine aspects of circumventricular organs. In Frontiers in Neuroendocrinology, W. T. Ganong and L. Martin, eds., pp. 3-32, Oxford University Press, New York.

Weiss, K. R., and I. Kupfermann (1978) Serotonergic neuronal activity and arousal of feeding in Aplysia californica. In Society for Neuroscience Symposia. Vol. 3: Aspects of Behavioral Neurobiology, J. A. Ferrendelli, ed., pp. 66-89, Society for Neuroscience, Bethesda, MD.

Weiss, K. R., M. Schonberg, D. E. Mandelbaum, and I. Kupfermann (1978) Activity of an individual serotonergic neurone in Aplysia californica's synthesis of cyclic adenosine monophosphate. Nature 272: 727-728.

Weiss, K. R., D. E. Mandelbaum, M. Schonberg, and I. Kupfermann (1979) Modulation of buccal muscle contractility by serotonergic metacerebral cells in Aplysia: Evidence for a role of cyclic adenosine monophosphate. J. Neurophysiol. 42: 791-803.

Willard, A. L. (1980) Serotonin increases the probability that the leech nervous system will produce the swim motor program. Soc. Neurosci. Abstr. 6: 27. 